\title{
Use of artificial intelligence in last mile delivery
}

\author{
Peter Jucha ${ }^{1, *}$ \\ ${ }^{1}$ University of Zilina, Faculty of Operation and Economics of Transport and Communications, \\ Department of Communications, Univerzitna 1, 01026 Zilina, Slovakia
}

\begin{abstract}
.
Research background: Artificial intelligence is a term that is now known to almost everyone and is among the trends and innovations of Industry 4.0 for 2020. It is a much-discussed topic in the field of technology. Artificial intelligence and machine training are the driving forces across different industries. In many cases, artificial intelligence helps people in their work and simplifies it or even completely replaces the human workforce.

Purpose of the article: The purpose of the article is to state how artificial intelligence can affect and solve existing problems in last mile delivery. For example, inefficiency is a major problem with last mile delivery because the last section of delivery usually involves a number of shortdistance stops. However, a long waiting time for the customer to deliver the goods or incorrect allocation of resources and vehicles to the required areas can also be a problem. And it is artificial intelligence that should help solve such problems.

Methods: Comparison, Empirical and retrospective analysis are used within the analysis of different modes of last-mile delivery.

Findings \& Value added: The research results shows the ways in which artificial intelligence can help solve problems in last mile delivery. Examples include The Vehicle Routing optimization (VRO), which aims to calculate the most optimal delivery route or artificial intelligence technology, which is used to interpret various events, manage data, and apply predictive intelligence.
\end{abstract}

Keywords: Artificial intelligence; Last mile delivery; Logistics

JEL Classification: $O 31 ; O 32 ; O 33$

\section{Introduction}

\subsection{Logistic of "last mile"}

The final part of the supply chain - „last mile” of transport of a particular product to the final customer or to a particular point of sale, is often referred to as the most important element of the process of processing all orders. Distribution of orders for this ,last mile” is

*Corresponding author: peter.jucha@fpedas.uniza.sk 
the most important logistics process. Last mile logistics refers to this part of transit in supply chains, where the goods are delivered from the last point of transit to the end point or the place of delivery. It represents a part of logistics, which includes the direction of vehicles to physical distribution and thus plays a crucial role in ensuring the delivery of goods, in the right quantity and within predetermined deadlines, to customers. [1,2,3]

In many cases, logistics providers, face many challenges and problems in overcoming the last mile. Such challenges include: high order fulfillment costs for high price sensitive customers, strong competitive external pressure on free delivery services, increased customer expectations due to short delivery times, individually scheduled delivery times, the ability to track and customize specific shipments along with the possibility of choosing an alternative place of delivery of consignments as well as a large number of unsuccessful attempts to deliver or return products. The issues examined within the area of last mile transport of goods and cargo further include: review of delivery strategies, delivery options, environmental impacts and humanitarian aid. [1,2]

The logistics industry is coping with many challenges and problems at the same time, and this is forcing the industry, in addition to developing innovations, to solve things in new ways. Logistics service innovations apply to basic as well as complex logistics services. Specific examples of logistics innovations in recent decades include: electronic data interchange (EDI), cross-docking, radio frequency identification (RFID) and joint planning, forecasting and supplementation (CPFR). However, new innovations, models and concepts are also being implemented specifically as part of last mile delivery. The rapid growth and development of cities forces the optimization of distribution processes through efficient last mile logistics models. The development of urban logistics vehicles (ULV) with low fuel consumption and high efficiency is expected. In addition, the ever-increasing volume of packages that need to be delivered to end customers every day has meant that many new last mile concepts have been introduced in recent years. The reason for which these new concepts were introduced was caused by the trend of increasing number of orders, which have a negative impact on the environment and safety, as more and more vans are needed to cover the last mile towards the customer. New last mile concepts include: deliveries using drones or small ground autonomous robots launched from trucks, distribution of goods by electric vehicles, deliveries to the trunk of parked cars, combined distribution of freight transport with public and private vehicles, reverse logistics, crowdsourcing of deliveries or mobile lockable cabinets. $[4,5,6]$

With the growing digitalization of the logistics industry, more and more companies are also starting to use artificial intelligence. They use it in their logistics and supply chain to reduce the amount of time and money they have to spend on finding out how, when and where to deliver a good or service. [7]

\subsection{Artificial intelligence}

The long process of automation of various business processes resulted in the introduction of artificial intelligence. This technology has made it possible to increase productivity and support economic growth. Artificial intelligence (AI) is an intelligence, which performs automated manual work of intelligent machines that can function like humans in certain respects. If we take into account the human perspective AI is also a simulation of human intelligence by machines programmed to think like humans. These machines have to be able to perform cognitive functions similar to human intelligence to mimic human actions.

However, it is also a designation that can be used with any machine that exhibits characteristics associated with the human mind, such as learning or problem solving. Key parts of the concept of AI consider the abilities of rationalization and action, that the 
greatest chance of achieving a specific goal, as an ideal characteristic associated with AI. $[8,9]$

There are two directions of AI research. The first is a technical one. Technical direction follows new methods, architectures and technologies that make it possible to constantly improve and refine AI and push the overall boundaries of machine intelligence. The second one focuses on issues related to implementation of AI technology. Thanks to this research dedicated to implementation of AI, the human understanding of how people can make the best use of AI in the socio-technical world, in which this technology must work, is broadened. In the case of AI, there are several different forms of learning. The simplest way is learning based on the trial and error. An example can be the application of past experience to analogous new situations. $[10,11]$

AI technology can be applied in various industries. Commonly the AI is being tested and used in the medical industry. Other examples of machines that use AI include computers that play chess or computers that autonomously drive cars and other vehicles. Each of these machines must consider the consequences of all its actions, because each of them has an impact on the final output. For example, in the case of autonomous vehicles, the computer system takes into account all received external data and at the same time calculates them so that the whole system works without causing a collision. [9]

The growth of new technologies, such as AI, various intelligent technologies, automation and robotics, has and will have a significant impact on all aspects of human society and economy. Many such changes will undoubtedly improve people's life, but the overall nature of the expected impact of the digital revolution on people's work and employment is controversial. For example, a reduction in labor demands significant qualitative changes in the skills provided by the workforce. Nevertheless, AI technologies provide great opportunities for new products, new services and productivity improvements. New technologies that are tied to AI will eventually become an essential part of businesses and entire economies. In the case of AI, progress is constantly being made because these systems are able to learn and improve. They make much more accurate predictions and are able to perform tasks such as navigation, which until recently was the exclusive domain of people's expertise. [12, 13]

\section{Methodology}

The main goal of this article is to identify and analyze how AI helps and solves last mile delivery problems and how this process helps to make it more efficient. To achieve this goal, it was necessary to use methods such as comparison, empirical and retrospective analysis of secondary sources that deal with this topic.

Empirical analysis, or otherwise empirical research, is research that is based on experience. Specifically, it is the collection of primary or secondary data by specific researchers. For the purpose of this article, secondary data dealing with the topic of AI and its primary use in the field of last mile delivery were analyzed. Some of the data were already included in the article on the basis of previous experience with the researched topic. The sources analyzed were mainly articles and contributions from the Internet, which deal with the topic of current innovations in the field of last mile delivery. From these articles, that were examined, those were selected that spoke specifically about the use of AI in last mile delivery. There were a number of sources on the Internet that talked about last-mile delivery innovations. It is a topic that changes every year. New problems and challenges arise, as well as new ways, methods and technologies that allow them to be solved. The number of Internet resources dealing with this topic has been increasing in recent years. In addition to Internet resources, contributions and scientific articles from the Web of Science 
database dealing with the topic were also analyzed, but their number was significantly lower compared to Internet resources, that were used.

Retrospective analysis, or otherwise retrospective research, is used when the outcome of specific events is already known. For the purposes of the article, the ways of using AI in the field of last mile delivery were analyzed in order to determine the reasons for which specific technologies or methods were developed. Based on these data, the theoretical part of the article was processed. In this part, it is stated, for example, that the problems that can be encountered when delivering to the last mile, were the deadlines for delivery of goods, the possibility of tracing and checking orders and so on. To this end, contributions and scientific articles from the Web of Science database dealing with the topic of the last mile were analyzed. This is still a new issue because almost all of the cited posts were only a few months old.

A comparison method is a method designed to search for objects that have some feature or features in common. It is used to determine the agreement and differences between the examined objects. For the purpose of the article, the method of comparison was used only to compare the individual information in the analyzed sources, whether they match or are significantly different.

In order to provide definitions of basic terms and the theoretical background of the article, mainly scientific articles from the Web of Science database were analyzed.

The results of the article were processed on the basis of secondary research. Within it, mainly internet sources were analyzed, which provided the information necessary to fulfill the main goal of the article.

\section{Results}

Based on the analyzed data from secondary sources, this section lists some ways in which $\mathrm{AI}$ is used in the field of last mile delivery and logistics. The results show, in particular, the ways to solve the main problems of last mile delivery mentioned in the theoretical part of this article. The individual subchapters of this chapter are divided according to the specific use of AI in the area of last mile delivery.

The share of last mile delivery costs represents $53 \%$ of the total transport costs. However, last mile delivery is becoming less demanding due to the use of logistics and route planning algorithms that are powered by AI. The AI in last mile delivery, in terms of its importance:

- allows to accurately manage even the most complex set of data,

- allows the use of data systems to create data sets used to control patterns and various phenomena,

- $\quad$ produces data models that focus on predictive analytics. [14]

The use of AI in last mile coordination and delivery has a positive impact on last mile logistics. Because AI performs powerful calculations and machine learning. It tracks and then analyzes historical trends, and predicts certain patterns based on the findings. Delivery options could be combined with a AI performed analysis of the situation to determine, which specific order should be processed and delivered as efficiently as possible. AI also can help to direct supply more efficiently, which is ultimately the key to profitability. In addition, it could help retailers and their shipping partners in particular to better predict shipments based on the historical data, which could significantly help delivery services to plan deliveries in advance. Thanks to this, they could better overcome the fluctuations between the Black Friday period and the „dead zone”, which marks the center of February. [15] 


\subsection{The Vehicle Routing optimization}

At present, the Vehicle Routing optimization (VRO) is still one of the important topics which, if more examined, could be used to reduce environmental pollution. The VRO is represented by many AI applications, with the aim of designing the lowest cost routes to meet customer demand, including the solution of the shortest route problem, the traveling salesman problem and the driving problem. The complexity of all operations varies according to specific customers. The main essence of VRO is to calculate a route under specific conditions, which ultimately minimizes the total cost of transport, by reducing, for example, the total distance traveled, the number of vehicles used or the total time of transport. $[7,16,17]$

VRO help reduce the cost of last mile services by optimizing routes and allocating resources. The resulting savings can be significant because more than half of the costs associated with a typical logistics company are covered in the implementation of the first or last mile. [7]

VRO can be classified as single-vehicle and multi-vehicle. [7]

Table 1. Classification of VRO.

\begin{tabular}{|c|c|}
\hline Single-vehicle & Multi-vehicle \\
\hline one vehicle with one depot & more vehicles with more depots \\
\hline one endpoint & multiple endpoints \\
\hline $\begin{array}{c}\text { Finding the right order of addresses to } \\
\text { provide services with minimal cost. }\end{array}$ & $\begin{array}{c}\text { Finding the optimal route for all vehicles with given } \\
\text { trade restrictions at minimal cost. }\end{array}$ \\
\hline
\end{tabular}

\subsection{Last Mile Platform}

Data from various sources can be used to optimize the route. This includes transport information, traffic patterns, GPS data or weather information. Such data collection can significantly affect fuel, personnel or other overhead costs associated with last mile delivery. A good example of a technology that allows delivery routes to be optimized in this way is an AI-based logistics platform called the Last Mile Platform (LaMP) developed by the Singaporean delivery company SingPost. LaMP combines various last mile delivery services such as couriers, collection points for brick-and-mortar stores or consignment boxes into one universal platform, regardless of the variability of technologies used by different courier companies. LaMP also uses AI to automate courier routes depending on a variety of factors, including parcel delivery destinations, real-time traffic data, or weather data. The software is also able to inform all parties involved about the movement of couriers. This is a feature that allows customers to be notified 30 minutes before the order is delivered that the order is almost at its destination. All the mentioned factors and possibilities can be operated through a single screen called ,the control tower”, which provides comprehensive visibility connecting a number of GPS and API tracking systems. $[18,19,20]$

\subsection{Autonomous things}

The trend in the field of logistics services is currently the automation of activities related to the distribution of consignments, namely due to the growing volume of consignments 
originating from e-commerce. Autonomous things are also a good example of the use of AI in last mile delivery. These are physical devices that use AI to automate various tasks previously performed by humans. However, autonomous delivery does no longer need any human intervention during its entire process. AI and machine learning allow autonomous delivery to be as fast and secure as possible. Many delivery companies are testing and developing autonomous delivery facilities that provide logistics solutions that help improvise mobility, vehicle capacity and reduce delivery time. Retailers are able to deliver orders to customers on the same day they receive them. Examples of such autonomous devices are robots, drones and various autonomous vehicles, ships or other devices. The automation of these devices goes beyond the capabilities of hard-coded models. These devices use AI to control advanced responses to more natural interactions with the environment and humans. Autonomous vehicles will be increasingly used in the field. [21, 22, 23]

Autonomous vehicles and autonomous driving are already a very discussed topic of today. Full autonomy, which represents the fifth level of autonomy, has not yet been realized. Some Tesla cars can currently be switched to autopilot mode when driving on the motorway, but this is only possible up to second level, which is driving support, or up to third level, where such a vehicle is operated by the driver only in an emergency. [24]

Many delivery companies have already started using aerial drones that deliver the order directly to the customer. However, certain challenges associated with the use of drones still need to be overcome. Drones will be used primarily to deliver orders to locations that are difficult to access. The development of aerial drones is considered to be an effective method of securing the growing demands of customers related to the delivery of orders directly to them. For this reason, a large increase and extension of autonomous delivery to the last mile is expected. [21]

\section{Discussion and Conclusion}

New technologies strengthen the perception of companies' activities at several levels of sustainability, which can be economic, environmental and social. They are linked to tackling climate change, but also to the degree of trust between stakeholders.

Decisions on the implementation of the latest technologies, innovations and new business models concern the solution of open/closed, free/charged, cooperation/competition. These decisions determine the application of new technologies and business models in the scope and time with regard to market creation, the size and sustainability of the ecosystem, the platform's ability to generate and exploit network effects. [25]

These interactions are becoming particularly important in large large-scale network systems. These include industries based on a network technology structure. The application of new technologies in these companies increases their value and competitiveness. If the investment in technologies is in line with the strategic direction of the company, the conditions are created for influencing performance and overall productivity, further innovations in products and maintaining a competitive advantage.

Technologies and software that work on the principle of AI are proving to be very helpful in last mile delivery. They streamline the delivery of orders to customers and save costs by optimizing the entire delivery process. Technology such as a drone can reach places that are otherwise difficult to access. Autonomous cars, in turn, reduce delivery times. At the same time, it is possible to see how machines and technologies are slowly replacing people. And due to the ever-increasing demands of customers to deliver their orders, it is to be expected that this trend will continue to grow. But the question is, how long will human participation be needed in these technologies? In other sectors, it is 
announced that people will lose their jobs due to the introduction of new technologies and mainly due to AI. Will this also be the case of logistics?

It is clear that new technologies and software with the support of AI will be constantly developing. There will be more and more autonomous devices on the road and in the air. At present, they are not yet at a level where they could function completely independently without human intervention, but it is only a matter of time before development enters a phase of complete autonomy. However, with full autonomy, will there be no accidents? Will there be no damage that will cause more problems than benefits?

In addition to autonomous vehicles and equipment, more and more new technologies and applications are being used in the field of last mile delivery, which are created primarily to optimize delivery routes, reduce the cost of the delivery process as well as reduce the number of deliverers and the like. Examples include Vehicle Routing optimization (VRO) applications and the Last Mile Logistics Platform (LaMP), which combines various last mile delivery services. Again, these are the concepts that are gradually replacing people's activities, such as delivery route planning.

In general, it can be argued that AI-based technologies simplify and streamline a number of last mile delivery activities and operations, but at the same time pose a certain threat to the jobs of people whose activities are able to replace.

This contribution was supported by the project VEGA 1/0152/18 Business models and platforms in digital space.

\section{References}

1. Janevic, M., Wikenbach, M., (2020). Characterizing urban last-mile distribution strategies in mature and e,erging e-commerce markets. Transportation Research Part A: Policy and Practice, 133(2020), 164-196.

2. Lu, SH., Suzuki, Y., Clottey, T. (2020). The Last Mile: Managing Driver Helper Dispatching for Package Delivery Services. Journal of Business Logistics, 41(3), 206221.

3. Ko, S. Y., Sari, R. P., Makhmudov, M., Ko, C. S. (2020). Collaboration Model for Service Clustering in Last-Mile Delivery. Sustainability, 12(14), 5844.

4. Schwerdfeger, S., Boysen, N. (2020). Optimizing the changing locations of mobile parcel lockers in mile distribution. European Journal of Operational Research, 285(3), 1077-1094.

5. Wang, X. Q., Yuen, K. F., Wong, Y. D., Teo, C. C. (2020). E-consumer adoption of innovative last-mile logistics services: A comparison of behavioural models. Total Quality Management and Business Excellence, 31(11-12), 1381-1407.

6. Urzua-Morales, J. G., Sepulveda-Rojas, J. P., Alfaro, M., Fuertes, G., Ternero, R., Vargas, M. (2020). Logistic Modeling of the Last Mile: Case Study Santiago, Chile. Sustainability, 12(2), 648.

7. Open Data Science. (2019, October 14). AI as the Ultimate Disrupter in Logistics: How to Manage Last-Mile Costss? Medium. Retrieved from: https://medium.com/@ODSC/ai-as-the-ultimate-disrupter-in-logistics-how-to-managelast-mile-costs-c4874e8f2ea0

8. Goyal, A., Aneja, R. (2020). Artificial intelligence and income inequality: Do technological changes and worker's position matter? Journal of Public Affairs, 20(3), $1-10$. 
9. Frankenfield, J. (2020, March 13). Artificial Intelligence (AI). Investopedia. Retrieved from: https://www.investopedia.com/terms/a/artificial-intelligence-ai.asp

10. Coiera, E. (2019). The Last Mile: Where Artificial Intelligence Meets Reality. Journal of Medical Internet Research, 21(11), 1-4.

11. Copeland, BJ. (2020, August 11). Artificial intelligence. Britannica. Retrieved from: https://www.britannica.com/technology/artificial-intelligence

12. Rodriguez-Bustelo, C., Batista-Foguet, J. M., Serlavos, R. (2020). Debating the Future of Work: The Perception and Reaction of the Spanish Workforce to Digitization and Automation Technologies. Frontiers in Psychology, 11, 1-14.

13. Hemphill, T. A. (2019). Prediction Machines: The Simple Economics of Artificial Intelligence, Boston. Journal of General Management, 45(1), 50-51.

14. Telluru, P. (2019, November 22). How AI is Transforming Last Mile Delivery. ShipConsole. Retrieved from: https://shipconsole.com/shipping-automation-last-miledelivery/

15. Meade, J. (2019. February 7). How AI Could Help Solve the Last Mile Delivery Problem. Medium. Retrieved from: https://medium.com/datadriveninvestor/how-aicould-help-solve-the-last-mile-delivery-problem-f01823d0cd30

16. Tu, W., Li, Q. Q., Fang, Z. X., Zhou, B. D. (2015). A Novel Spatial-Temporal Voronoi Diagram-Based Heuristic Approach for Large-Scale Vehicle Routing Optimization with Time Constraints. ISPRS International Journal of Geo-Information, 4(4), 20192044.

17. Kedia, R. K., Naick, B. K. (2017). Review of Vehicel Route Optimisation. In Proceedings of the 2nd international conference on intelligent transporatation engineering (ICITE) (pp. 57-61). Singapore: Singapore.

18. How AI Can Improve Sustainability in Last-Mile Delivery. Transmetrics. Retrieved from: https://transmetrics.eu/blog/how-artificial-intelligence-can-improvesustainability-in-last-mile-delivery/

19. Norman, H. (2018, December 17). SingPost integrates LogiNext's AI to LaMP logistics platform. ParcelandPostalTechnologyInternational. Retrieved from: https://www.parcelandpostaltechnologyinternational.com/news/technology/singpostintegrates-loginexts-ai-to-lamp-logistics-platform.html

20. Urquhart, D. (2018, December 11). SingPost integrates AI into its Southeast Asia-wide logistics platform. Asiacargobuzz. Retrieved from: https://asiacargobuzz.com/2018/12/11/singpost-integrates-ai-into-its-southeast-asiawide-logistics-platform/

21. Abraham, Aji. (2020, January 16). How Technology is Improving Last-Mile Delivery in 2020. Mystory. Retrieved from: https://yourstory.com/mystory/technology-improvinglast-mile-delivery-2020

22. KPC-Group, s.r.o. (2019, November 11) Top 10 strategických technologických trendov pre rok 2020. Pcrevue. Retrieved from: https://www.pcrevue.sk/a/Top-10strategickych-technologickych-trendov-pre-rok-2020

23. Madlenakova, L., Matuskova, M., Madlenak, R., Rudawska, A., Rybicka, I. (2018). Solutions of the Roller Conveyor in Terms of Logistics Provider. Advances in Science and Technology-research Journal, 12(4), 1-9.

24. Uzzaman, A. (2019, December 30). 10 technology trends that will impact our lives in 2020. VentureBeat. Retrieved from: https://venturebeat.com/2019/12/30/10technology-trends-that-will-impact-our-lives-in-2020/ 
25. Corejova, T., Al Kassiri, M. (2016). Knowledge as the Key to the Global Cooperation and Its Important Role Among Nations. In G. Lee (Ed.), Proceedings of the 3rd International Conference on Power and Energy Systems PES 2016 (pp. 181-183). Thailand: Bangkok. 